\title{
Some Remarks on Commuting Fixed Point Free Automorphisms of Groups
}

\author{
Akhavan-Malayeri M.; \\ Department of Mathematics, Alzahra University \\ Received: 18 Nov 2013 \\ Revised: 10 Nov 2014
}

\begin{abstract}
In this article we will find necessary and sufficient conditions for a fixed point free automorphism (fpf automorphism) of a group to be a commuting automorphism. For a given prime $\mathrm{p}$, we find the smallest order of a non abelian p-group admitting a commuting fixed point free automorphism. We prove that a group of order $\mathrm{p}^{3}$ having a commuting fpf automorphism, has a restricted structure. Moreover, we prove that if a finite group G admits a fpf automorphism of order 4, then the converse of Laffey's result holds in $\mathrm{G}^{1}$.
\end{abstract}

Keywords: Commuting automorphisms, Fixed point free automorphisms, p-groups.

\section{Introduction}

Let $G$ be a group. By $Z(G), G^{\prime}$, Aut $(G)$, $\operatorname{Aut}_{c}(G), \operatorname{Inn}(G)$ and $\gamma_{i}(G)$ we denote the ce nter, the commutator subgroup, the group of all automorphisms, the group of central aut omorphisms, the group of all inner automorphisms and the ith term of the lower central series of G, respectively.

An automorphism $\alpha$ of $G$ is called a fixed point free automorphism (fpf automorphis m) if $\alpha$ fixes only the identity of G. A subgroup $H \leq$ Aut $(G)$ is said to be fpf if every non-trivial automorphism of $\mathrm{H}$ is fpf.

It is well known that a finite group $G$ admits a fpf automorphism of order two if and only if G is abelian of odd order (see [2]). By a famous theorem of J. G. Thomson [12], a finite group admitting a fpf automorphism of prime order must be nilpotent.

An automorphism $\alpha$ of $\mathrm{G}$ is called a commuting automorphism if $\mathrm{g} \alpha(\mathrm{g})=\alpha(\mathrm{g}) \mathrm{g}$, for all $\mathrm{g} \in \mathrm{G}$. We denote the set of all commuting automorphisms of $\mathrm{G}$ by $\mathcal{A}(\mathrm{G})$. It seems tha $\mathrm{t}$ for the first time, the commuting automorphisms are defined, in various rings(see [1], [5] and [10]). Deaconsecu, Silberberg and Walls in [3] showed that even though $\mathcal{A}(\mathrm{G}) \mathrm{h}$ as a number of group properties, it does not necessarily form a subgroup of Aut(G).

\footnotetext{
*2010 Mathematics Subject Classification: Primary 20F28, Secondary 20D45
}

*Corresponding author: mmalayer@alzahra.ac.ir 
A group $\mathrm{G}$ is called $\mathcal{A}(\mathrm{G})$-group if the set $\mathcal{A}(\mathrm{G})$ forms a subgroup of $\operatorname{Aut}(\mathrm{G})$. In [13] we proved that the minimum order of a non- $\mathcal{A}(\mathrm{G})$, p-group is $\mathrm{p}^{5}$. We also found the $\mathrm{s}$ mallest group order of a non $\mathcal{A}(\mathrm{G})$, p-group. Furthermore, we proved that for any prime $\mathrm{p}$ and for all integers $\mathrm{n} \geq 5$, there exists a non- $\mathcal{A}(\mathrm{G})$, p-group of order $\mathrm{p}^{\mathrm{n}}$.

The commuting automorphisms fix $\gamma_{3}(G)$ pointwise. Obviously, the group of central automorphisms of $\mathrm{G}$ is contained in the set of all commuting automorphisms. The commuting automorphisms have certain interesting properties close to those of central automorphisms. The relationship between commuting automorphisms and central automorphisms discussed in [3]. Further, the authors in [4] have proved that the inner automorphisms which belong to $\mathcal{A}(\mathrm{G})$ are precisely the inner automorphisms which are induced by 2-Engel elements.

In this article, we will find necessary and sufficient conditions for a fpf automorphism to be a commuting automorphism. We also study the following question which naturally arises in this regard.

"What is the smallest order of a non-abelian p-group, admitting a commuting fpf aut omorphism?"

To answer this question, we will classify all groups of order $\mathrm{p}^{3}$ which have a commuting fpf automorphism. We prove that a group of order $\mathrm{p}^{3}$ having a commuting fpf automorphism, has a restricted structure:

Theorem 1. Let $\mathrm{G}$ be a group of order $\mathrm{p}^{3}$. Then $\mathrm{G}$ has a commuting fpf automorphism if and only if $\mathrm{G}$ is of one of the following types:

(i) an elementary abelian group of order 8;

(ii) an abelian group of odd order;

(iii) an extra-especial p-group of exponent $\mathrm{p}, \mathrm{p} \geq 5$.

In particular all groups of type (i) or (ii) have a commuting fpf automorphism of order 2. If $\mathrm{G}$ is of type (iii) and $\mathrm{p}$ is a Fermat prime number then $\mathrm{G}$ has a commuting fpf autom orphism of order $\mathrm{p}-1$.

As an immediate consequence of the above theorem, we obtain the following result:

Corollary 2. For a given prime $\mathrm{p}$, the minimum order of a non-abelian p-group, having a commuting fpf automorphism is 125 .

We continue by recalling a few results and notations concerning a finite group $G$ which admits a commuting fpf automorphism $\alpha$ of order 4 (see [6]). 
First of all G is of odd order. The set of elements of G left fixed by $\alpha^{2}$ is an $\alpha$-invariant subgroup of $G$, which is denoted by $F$. If $F \neq 1$, the restriction of $\alpha$ to $F$ is a fpf automo rphism of $F$, which is of order 2. Hence $F$ is abelian and $\alpha(f)=f^{-1}$, for all $f$ in $F$. The $s$ et of all $\mathrm{h}$ in $\mathrm{G}$ for which $\alpha^{2}(\mathrm{~h})=\mathrm{h}^{-1}$ is denoted by I. The set I need not be a subgroup of $G$ and $G=F I=I F$. We will use these notations for the case $|\alpha|=4$.

Furthermore we recall that if $\alpha$ is a fpf automorphism of order $n$ of a finite group $G, t$ hen $\mathrm{x}^{1+\alpha+\cdots+\alpha^{\mathrm{n}-1}}=1$.

By using the above notations, the following result displays the necessary and sufficient conditions for a fpf automorphism to be a commuting automorphism.

Theorem3. Let $\alpha$ be a fpf automorphism of a finite group $G$ with $\alpha$ of order $n$.

(i) If $\mathrm{n} \leq 3$, then $\alpha$ is a commuting automorphism.

(ii) If $\mathrm{n}=4$, then $\alpha$ is a commuting automorphism if and only if $[\mathrm{f}, \alpha(\mathrm{h})]=$ $[\alpha(f), h]$, for all $h \in I, f \in F$.

(iii) If $\mathrm{n} \geq 5$, then $\alpha$ is a commuting automorphism if and only if

$$
x^{1+\alpha+\cdots+\alpha^{n-3}}=x^{\alpha^{n-3+\cdots+\alpha+1}},
$$

for all $\mathrm{x} \in \mathrm{G}$.

The next result from [8], is useful for many computations.

Theorem (T. J. Laffey) If $\alpha \in \mathcal{A}(\mathrm{G})$ and $\mathrm{x}, \mathrm{y} \in \mathrm{G}$, then $[\alpha(\mathrm{x}), \mathrm{y}]=[\mathrm{x}, \alpha(\mathrm{y})]$.

As a consequence of the above theorem, we obtain that if a finite group $G$ admits a fpf a utomorphism $\alpha$ of order 4 , then the converse of Laffey's result holds for $\alpha$.

In the following result we find the structure of a finite group having a central fpf automorphism.

Theorem4. Let $\mathrm{G}$ be a finite group. Then $\mathrm{G}$ has a central $\mathrm{fpf}$ automorphism if and only if $G$ is an abelian group and $|G|$ is odd or $G \cong A \times B$, where $B$ is a group of odd order an $\mathrm{d} A$ is the Sylow 2 -subgroup of $\mathrm{G}$ whose invariants are

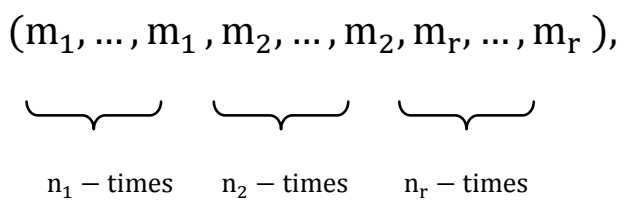

where $0<\mathrm{m}_{1}<\mathrm{m}_{2}<\cdots<\mathrm{m}_{\mathrm{r}}$ and $\mathrm{n}_{\mathrm{i}}>1$ for all $\mathrm{i}$.

\section{Proof of the main results}

Thoughout the paper we use the standard notations from [11]. In particular, if $x, y \in G$ then $x^{y}=y^{-1} x y$ and $[x, y]=x^{-1} y^{-1} x y$. 
Proof of Theorem1. It is clear that if $p>2$, then every abelian group of order $\mathrm{p}^{3}$ admit $\mathrm{s}$ a commuting fpf automorphism. Suppose $\mathrm{G}$ is an abelian group of order 8. It follows fr om [7, Theorem 4.2] that $\mathrm{G}$ admits a fpf automorphism if and only if $\mathrm{G}$ is an elementary abelian group. Hence we get the group (i) in the theorem. Thus we may assume that G is not abelian. If $\mathrm{G}$ is a non-abelian group of order $2^{3}$, then it is easy to show that Aut $_{c}(G)=\mathcal{A}(G) \simeq \mathbb{Z}_{2} \times \mathbb{Z}_{2}$ and $\mathrm{Z}(\mathrm{G})=\mathrm{G}^{\prime} \simeq \mathbb{Z}_{2}$. Hence $\mathrm{G}$ does not admit a fpf autom orphism.

Let $G$ be an extra-especial $p$-group of order $p^{3}, p \geq 3$. If $G$ has exponent $p^{2}$, then Aut $_{c}(G)=\mathcal{A}(G) \simeq \mathbb{Z}_{2} \times \mathbb{Z}_{2}$ (see [9]). Hence $G$ does not admit a commuting fpf automorphism.

Now we may assume $G=<x, y, z \mid x^{p}=y^{p}=z^{p}=1,[x, z]=[y, z]=1,[x, y]=z>$, the extra-especial p-group of exponent p. In [9] we defined $\theta_{\mathrm{kst}}$, where $1 \leq \mathrm{k} \leq \mathrm{p}-1$ and $0 \leq \mathrm{s}, \mathrm{t} \leq \mathrm{p}-1$, to be the automorphism of $\mathrm{G}$ sending $\mathrm{x}$ to $\mathrm{x}^{\mathrm{k}} \mathrm{z}^{\mathrm{s}}, \mathrm{y}$ to $\mathrm{y}^{\mathrm{k}} \mathrm{z}^{\mathrm{t}}$ and $\mathrm{z} \mathrm{t}$ $\mathrm{o} \mathrm{z}^{\mathrm{k}^{2}}$.

We also proved that $\mathcal{A}(\mathrm{G})=\left\{\theta_{\mathrm{kst}} \mid 1 \leq \mathrm{k} \leq \mathrm{p}-1,0 \leq \mathrm{s}, \mathrm{t} \leq \mathrm{p}-1\right\}$, which is a nonabelian group of order $\mathrm{p}^{2}(\mathrm{p}-1)$. In particular $\mathcal{A}(\mathrm{G}) \neq \operatorname{Aut}_{\mathrm{c}}(\mathrm{G})$, and $\operatorname{Aut}_{\mathrm{c}}(\mathrm{G}) \simeq$ $\mathbb{Z}_{\mathrm{p}} \times \mathbb{Z}_{\mathrm{p}}$

If $\mathrm{p}=3$ and $\alpha=\theta_{\mathrm{kst}} \in \mathcal{A}(\mathrm{G})$, then $\alpha(\mathrm{z})=\mathrm{z}$. Hence $\mathrm{G}$ does not admit a commuting fpf automorphism.

Finally, we may assume that $\mathrm{p} \geq 5$. Let $\mathrm{k}(1 \leq \mathrm{k} \leq \mathrm{p}-1)$ be a primitive root modulo $\mathrm{p}$, and let $\alpha=\theta_{\text {koo }} \in \mathcal{A}(\mathrm{G})$. Then $\alpha$ sends $\mathrm{x}$ to $\mathrm{x}^{\mathrm{k}}, \mathrm{y}$ to $\mathrm{y}^{\mathrm{k}}$, and $\mathrm{z}$ to $\mathrm{z}^{\mathrm{k}^{2}}$. We shall prove that $\alpha$ is a fpf automorphism. If $\mathrm{g}=\mathrm{x}^{\mathrm{v}} \mathrm{y}^{\mathrm{u}} \mathrm{z}^{\mathrm{l}} \in \mathrm{G}$, where $0 \leq \mathrm{v}, \mathrm{u}, \mathrm{l} \leq \mathrm{p}^{-1}$, then $\alpha(\mathrm{g})=\mathrm{g}$ if and only if $\mathrm{x}^{\mathrm{vk}} \mathrm{y}^{\mathrm{uk}} \mathrm{z}^{1 \mathrm{k}^{2}}=\mathrm{x}^{\mathrm{v}} \mathrm{y}^{\mathrm{u}} \mathrm{z}^{\mathrm{l}}$.

Hence modulo $\mathrm{Z}(\mathrm{G}), \mathrm{x}^{\mathrm{vk}} \mathrm{y}^{\mathrm{uk}}=\mathrm{x}^{\mathrm{v}} \mathrm{y}^{\mathrm{u}}$. Recall that $\mathrm{G} / \mathrm{Z}(\mathrm{G})$ is an elementary abelian group. Hence $\mathrm{vk} \equiv_{\mathrm{p}} \mathrm{v}$ and $\mathrm{uk} \equiv_{\mathrm{p}} \mathrm{u}$. Therefore $\mathrm{v}=\mathrm{u}=0$ and $\mathrm{z}^{\mathrm{lk}^{2}}=\mathrm{z}^{\mathrm{l}}$. So $\mathrm{lk}^{2} \equiv_{\mathrm{p}} \mathrm{l}$ and $\mathrm{l}=0$. Thus $\mathrm{g}=\mathrm{x}^{\mathrm{v}} \mathrm{y}^{\mathrm{u}} \mathrm{z}^{\mathrm{l}}=1$ and $\alpha$ is a commuting fpf automorphism of G. Hence we get the group (iii) in the theorem.

It is clear that the groups of type (i) or (ii) have a commuting fpf automorphism of order 2. Finally, assume $G$ is of type (iii) and $p=F_{n}=2^{2^{n}}+1$ is a Fermat prime number. Let $\alpha=\theta_{\text {koo }} \in \mathcal{A}(\mathrm{G})$, where $\mathrm{k}$ is a primitive root modulo $\mathrm{p}$. Now by Fermat's Theorem $\mathrm{k}^{\mathrm{p}-1} \equiv_{\mathrm{p}} 1$, so it follows that $\alpha^{\mathrm{k}^{\mathrm{p}-1}}(\mathrm{x})=\mathrm{x}$ and $\alpha^{\mathrm{k}^{\mathrm{p}-1}}(\mathrm{y})=\mathrm{y}$, but $\alpha^{\mathrm{p}-1}(\mathrm{z})=$ $\mathrm{z}^{\mathrm{k}^{2^{\mathrm{p}-1}}}$ and hence $\mathrm{p}-1 \mid 2^{\mathrm{p}-1}$. Therefore $2^{\mathrm{p}-1}=\mathrm{m}(\mathrm{p}-1)$, for some $\mathrm{m} \in \mathbb{N}$. 
This implies that $\mathrm{k}^{2^{\mathrm{p}-1}}=\left(\mathrm{k}^{\mathrm{p}-1}\right)^{\mathrm{m}} \equiv_{\mathrm{p}} 1$ and $\alpha^{\mathrm{p}-1}(\mathrm{z})=\mathrm{z}^{\mathrm{k}^{2^{\mathrm{p}-1}}}=\mathrm{z}$. Thus $\alpha^{\mathrm{p}-1}(\mathrm{~g})=\mathrm{g}$, for any $\mathrm{g} \in \mathrm{G}$.

Moreover, $\alpha^{\mathrm{i}}(\mathrm{x}) \neq \mathrm{x}$ for any $\mathrm{i} \in\{1,2, \ldots, \mathrm{p}-2\}$. Therefore $|\alpha|=\mathrm{p}-1$ and this $\mathrm{c}$ ompletes the proof.

We continue by giving the proof of Theorem 3 .

Proof of Theorem3. (i) If $|\alpha|=2$, then $\mathrm{G}$ is abelian and $\alpha \in \mathcal{A}(\mathrm{G})=\operatorname{Aut}(\mathrm{G})$.

If $|\alpha|=3$, it follows from $[11,10.5 .1]$ that $\mathrm{x} \alpha(\mathrm{x}) \alpha^{2}(\mathrm{x})=1$ for all $\mathrm{x}$ in $\mathrm{G}$. Therefore $\mathrm{x}^{-1} \alpha\left(\mathrm{x}^{-1}\right) \alpha^{2}\left(\mathrm{x}^{-1}\right)=1$. It then follows that $\alpha(\mathrm{x}) \mathrm{x}=\alpha^{2}\left(\mathrm{x}^{-1}\right)=\mathrm{x} \alpha(\mathrm{x})$ and therefore $\alpha \in \mathcal{A}(\mathrm{G})$.

(ii) Let $\mathrm{n}=4$ and $\mathrm{g} \in \mathrm{G}$. Then, by [6, Lemma 1] there exists $\mathrm{f} \in \mathrm{F}$ and $\mathrm{h} \in \mathrm{I}$ such that $\mathrm{g}=\mathrm{fh}$. $[6$, Lemma 4$]$ implies that $[\alpha(\mathrm{h}), \mathrm{h}]=1$.

Now if $\alpha \in \mathcal{A}(\mathrm{G})$ then

$$
\begin{aligned}
{[\mathrm{f}, \alpha(\mathrm{h})] } & =\mathrm{f}^{-1}(\alpha(\mathrm{h}))^{-1} \mathrm{f} \alpha(\mathrm{h})=\alpha(\mathrm{f}) \alpha\left(\mathrm{h}^{-1}\right) \mathrm{fh}^{-1} \mathrm{~h} \alpha(\mathrm{h}) \\
& =\alpha\left(\mathrm{fh}^{-1}\right)\left(\mathrm{fh}^{-1}\right) \mathrm{h} \alpha(\mathrm{h})=\left(\mathrm{fh}^{-1}\right) \alpha\left(\mathrm{fh}^{-1}\right) \mathrm{h} \alpha(\mathrm{h}) \\
& =\mathrm{fh}^{-1} \alpha(\mathrm{f}) \alpha\left(\mathrm{h}^{-1}\right) \mathrm{h} \alpha(\mathrm{h}) \\
& =\alpha(\mathrm{f})^{-1} \mathrm{~h}^{-1} \alpha(\mathrm{f}) \mathrm{h} \\
& =[\alpha(\mathrm{f}), \mathrm{h}] .
\end{aligned}
$$

To prove the converse, we have $\left[\mathrm{f}, \alpha\left(\mathrm{h}^{-1}\right)\right]=\left[\alpha(\mathrm{f}), \mathrm{h}^{-1}\right]$.

Therefore, $\mathrm{f}^{-1} \alpha(\mathrm{h}) \mathrm{f}=\left[\mathrm{f}^{-1}, \mathrm{~h}^{-1}\right] \alpha(\mathrm{h})$. So we have

$$
\begin{gathered}
\alpha(\mathrm{g}) \mathrm{g}=\mathrm{f}^{-1} \alpha(\mathrm{h}) \mathrm{fh}=\left[\mathrm{f}^{-1}, \mathrm{~h}^{-1}\right] \alpha(\mathrm{h}) \mathrm{h}=\mathrm{fhf}^{-1} \mathrm{~h}^{-1} \alpha(\mathrm{h}) \mathrm{h} \\
=\mathrm{fh} \alpha(\mathrm{f}) \alpha(\mathrm{h})=\mathrm{g} \alpha(\mathrm{g}) .
\end{gathered}
$$

This implies $\alpha \in \mathcal{A}(\mathrm{G})$.

(iii) Let $\mathrm{n} \geq 5$. Then $\mathrm{x}^{1+\alpha+\cdots+\alpha^{\mathrm{n}-1}}=1$ for all $\mathrm{x}$ in $\mathrm{G}$. So we have $\mathrm{x}^{-1} \mathrm{x}^{-\alpha} \ldots \mathrm{x}^{-\alpha^{\mathrm{n}-1}}=1$. Therefore $\mathrm{x}^{\alpha} \mathrm{x}=\mathrm{x}^{-\alpha^{2}} \ldots \mathrm{x}^{-\alpha^{\mathrm{n}-1}}$ and we obtain

$$
\mathrm{x}^{\alpha} \mathrm{x}=\mathrm{xx}^{\alpha} \Leftrightarrow \mathrm{x}^{1+\alpha+\cdots+\alpha^{\mathrm{n}-3}}=\mathrm{x}^{\alpha^{\mathrm{n}-3}+\cdots+\mathrm{a}+1} .
$$

Finally, we turn to the proof of Theorem 4.

Proof of Theorem4. To prove if implication, let $\alpha$ be a nontrivial central fpf automorphism of $G$.Then $\alpha$ acts trivially on $G^{\prime}$. But, since $\alpha$ is fpf, we must have $\mathrm{G}^{\prime}=1$ and thus $\mathrm{G}$ is abelian. Therefore $|\mathrm{G}|$ is odd or $\mathrm{G}$ has a unique 2-Sylow subgroup A. Hence $|G|$ is odd or $G=A \times B$, where $B$ is an abelian group of odd order.

Now since $A$ is a characteristic subgroup of $G,\left.\alpha\right|_{A}$ is a fpf automorphism of $A$. Hence according to [7, Theorem 4.2], A has the desired invariants.

To prove only if implication, since $B$ is an abelian group of odd order, $B$ has a fpf automorphism $\beta$. Now [7, Theorem 4.2] implies that $A$ has a fpf automorphism $\alpha$. Define $\gamma: G \rightarrow G$ given by $\gamma(a b)=\alpha(a) \beta(b)$ for $a \in A$ and $b \in B$, then $\gamma$ is a fpf automorphism of $\mathrm{G}$. 
Obviously, according to Theorem 4, a finite group $\mathrm{G}$ has a central fpf automorphism if and only if $G$ is abelian and $|G| \equiv_{2} 1$ or $|G| \equiv_{4} 0$.

\section{Acknowledgments}

We thank the editors of the Journal of Mathematical Researches of Kharazmi University and the referee who have patiently read and verified this note, and also sugge sted valuable comments. The author also like to acknowledge the support of the Alzahra University.

\section{References}

1. Bell H. E., Martindale W. S., "Centralizing mappings of semiprime rings", Canad, Math. Bull. 30 (1987) 92-101.

2. Burnside W., "Theory of Groups of Finite Order", 2nd ed., Dover Publications Inc., New York (1955).

3. Deaconescu M., Silberberg G., Walls G. L., "On commuting automorphisms of groups", Arch. Math. (Basel) 79 (2002) 423-429.

4. Deaconescu M., Silberberg Gh., "Right 2-Engel elements and commuting automorphism of group", J. Algebra 238 (2001) 479-484.

5. Divinsky N., "On commuting automorphisms of rings", Trans. Roy. Soc. Canada, Sect. III. 49 (1955) 19-22.

6. Gorenstein D., Herstein I., "Finite groups admitting a fixed point free automorphism of order 4", Amer. J. Math. 83 (1961) 71-78.

7. Gross F., "Some remarks on groups admitting a fixed point free automorphism", Canad. J. Math. 20 (1968) 1300-1307.

8. Herstein I. N., Laffey T. J., Thomas J., "Problems and solutions: solutions of elementary problems: E3039", Amer. Math. Monthly 93 (1986) 816-817.

9. Kargarian Z., Akhavan-Malayeri M., "On the commuting automorphisms of groups of order $\mathrm{p}^{3}, "$ AAMS. (9) No. 2 (2011) 115-120.

10. Luh J., "A note on commuting automorphisms of rings", Amer. Math. Monthly 77 (1970) $61-62$

11. Robinson D. J. S., "A course in the Theory of Groups", Vol. (80) of Graduate Texts in Mat h. Springer-Verlag (2nd ed. 1996).

12. Thompson J., "Finite groups with fixed point free automorphisms of prime order", Proc. Nat. Acad. Sci. U.S.A. 45 (1959) 578-581.

13. Vosooghpour F., Akhavan-MalayerI M., "On commuting automorphisms of p-groups", Comm. Algebra, Vol. 41 (4) (2013) 1292-1299. 